\title{
Prognostic Significance of Combined Platelet Distribution Width and C-Reactive Protein Score in Esophageal Cancer
}

\author{
YUTA KAWAKITA ${ }^{1}$, SATORU MOTOYAMA ${ }^{1,2}$, YUSUKE SATO ${ }^{1}$, AKIYUKI WAKITA ${ }^{1}$, \\ YUSHI NAGAKI $^{1}$, KAZUHIRO IMAI $^{1}$ and YOSHIHIRO MINAMIYA ${ }^{1}$ \\ ${ }^{1}$ Department of General Thoracic Surgery, ${ }^{2}$ Department of Comprehensive Cancer Control, \\ Akita University Graduate School of Medicine, Akita, Japan
}

\begin{abstract}
Background/Aim: The platelet distribution width $(P D W)$ and serum $C$-reactive protein $(C R P)$ levels are known to be predictive of prognosis in various malignancies. Our aim was to determine whether combining PDW and serum CRP levels produces a prognostic indicator for esophageal cancer (EC) patients. Patients and Methods: A total of 168 EC patients who underwent neoadjuvant therapy prior to esophagectomy were included in this study. Results: We defined a combined PDW and CRP (CPC) score as follows: patients with both low pretherapeutic $P D W(\leq 12.4 \mathrm{fl})$ and high postoperative serum CRP levels $(\geq 0.5 \mathrm{mg} / \mathrm{dl})$ were assigned a score of 2, while patients with one or neither of those were assigned a score of 1 or 0 . A multivariable analysis showed that the CPC score was a significant risk factor for overall $(p=0.006)$ and recurrence-free $(p=0.004)$ survival. Conclusion: The CPC score is a strong prognostic indicator in EC patients.

Esophageal cancer (EC) is highly invasive and metastatic, making it one of the most malignant of gastrointestinal tumors (1). Over the course of therapy, EC patients frequently exhibit hematological and serological abnormalities due to the burdens of adjuvant therapy, highly invasive esophagectomy, and postoperative complications $(2,3)$. In recent years, much effort has gone into establishing a novel prognostic marker for EC, analogous to the neutrophil-to-lymphocyte ratio (NLR) (4), by combining hematological and serological parameters obtained from routine blood tests. The platelet distribution width (PDW), a parameter determined from
\end{abstract}

This article is freely accessible online.

Correspondence to: Yuta Kawakita, Department of General Thoracic Surgery, Akita University Graduate School of Medicine, 1-1-1 Hondo, Akita, 010-8543, Japan. Tel: +81 188846132, Fax: +81 188362615, e-mail: yutakawakita@gipc.akita-u.ac.jp

Key Words: Platelet distribution width, C-reactive protein, esophageal cancer. complete blood count data, is a marker of the heterogeneity of platelet size and reflects platelet activity more directly than the mean platelet volume (MPV) $(5,6)$. Activated platelets are recognized to be key players in tumorigenesis (7), and high PDW levels are reportedly related to poor outcomes in various types of cancer (8-12). On the other hand, a small number of studies have demonstrated that low PDW levels are significantly associated with poor prognoses in gastric cancer $(13,14)$ and lung cancer $(15,16)$.

$\mathrm{C}$-reactive protein (CRP) is one of the acute-phase proteins produced predominantly by hepatocytes in response to inflammation (17). Whether alone or combined with other routine blood data, elevated CRP levels have already been shown to be useful prognostic markers in several cancer types $(18,19)$. To the best of our knowledge, however, no studies have investigated whether combining PDW levels and serum CRP levels produces a stronger prognostic indicator of malignant tumors than other known prognostic markers. In the present study, therefore, we evaluated the predictive value of the combination of PDW levels and serum CRP levels for the survival of EC patients receiving neoadjuvant therapy (NAT) plus esophagectomy. In addition, we also determined at which point during the course of therapy each parameter correlated most strongly with prognosis.

\section{Patients and Methods}

Patients. This study was approved by the Ethics Committee of Akita University (Approval number: 547). It included 168 adult patients with cStage I-IVB EC who underwent NAT [21 neoadjuvant chemotherapy (NAC) and 147 NAC with radiotherapy (NACRT)] prior to esophagectomy at Akita University Hospital between September 2008 and December 2018. Cancer stage was classified according to the International Union Against Cancer tumor-node-metastasis (TNM) Classification of Malignant Tumors (8th edition) (20). Patients with Stage IVB had nonregional (lateral cervical) lymph node involvement but no distant organ metastasis. Informed consent for use of their clinical information in future medical research was obtained from all patients prior to surgery. The retrospective data were then collected for all patients at their initial visit (pretherapeutic time point), just before surgery 
(preoperative time point), and 2-3 months postoperatively (postoperative time point). In the measurement of hematological parameters, whole blood samples were collected in potassium ethylenediaminetetraacetic acid (EDTA)-containing tubes, and all samples were analysed within 30 min after blood collection using an automated hematological analyzer (Sysmex XS-800i, Kobe, Japan). Known prognostic markers, including the CRP to albumin ratio (CAR), controlling nutritional status (CONUT) score, lymphocyte-to-monocyte ratio (LMR), NLR, platelet-tolymphocyte ratio (PLR), prognostic nutritional index (PNI), and the modified Glasgow prognostic score (mGPS), were also measured at the three time points. The formulas used to calculate the scores for these prognostic markers are described elsewhere $(4,21-26)$.

Treatment strategy. In general, patients with locally advanced or lymph node-positive tumors underwent NACRT. For NACRT, the radiotherapy consisted of 40-41.4 Gy in 20-23 fractions. For NAT, the chemotherapy consisted of continuous infusion of 5-fluorouracil $\left(800 \mathrm{mg} / \mathrm{m}^{2} /\right.$ day) on days $1-5$, combined with cisplatin or nedaplatin $\left(80 \mathrm{mg} / \mathrm{m}^{2} /\right.$ day) on day 1 . This protocol was repeated twice with $3-$ to 6-week intervals in between. Our standard operative procedure consisted of transthoracic esophagectomy or video-assisted thoracoscopic esophagectomy with two- (mainly for abdominal EC patients) or three-field lymphadenectomy and reconstruction with a gastric tube or pedicled colon through the posterior mediastinal or subcutaneous route (27).

Statistical analysis. Values are expressed as the median (interquartile range). Differences between two groups were analyzed using the Wilcoxon rank sum test for continuous variables and the Pearson $\chi^{2}$ test or Fisher exact probability test for categorical variables. Survival length was determined from the date NAT was initiated to death or to the date of the last clinical followup. Survival curves were derived using the Kaplan-Meier method, and differences between the curves were assessed using the logrank test (the Bonferroni-Holm correction was utilized for pairwise multiple comparisons). The hazard ratios for mortality were determined using Cox's proportional hazards regression models. Variables added in multivariable analyses were determined with stepwise regression. Statistical analyses were performed using JMP 14.2 (SAS Institute, Cary, NC, USA). Values of $p<0.05$ (two-sided) were considered significant.

\section{Results}

Patient overview. The clinical characteristics of all patients included in this study are summarized in Table I. These patients included $145(86.3 \%)$ males and $23(13.7 \%)$ females with a median age of 64 years (range $=59-68$ years). Among the 168 patients, there were $6(3.6 \%)$ with adenocarcinoma and 162 with squamous cell carcinoma (96.4\%). The PDW levels at the pretherapeutic, preoperative, and postoperative time points were $11.1(10.2-12.4) \mathrm{fl}, 10.2$ (9.1-11.2) fl, and 9.9 (8.9-10.9) fl, respectively, while serum CRP levels at the three time points were $0.13(0.06-0.51) \mathrm{mg} / \mathrm{dl}, 0.14(0.06-$ $0.41) \mathrm{mg} / \mathrm{dl}$, and $0.13(0.05-0.46) \mathrm{mg} / \mathrm{dl}$, respectively. Interestingly, the PDW levels in our cohort tended to be lower than in earlier studies of esophageal cancer $(9,10)$.
Overall survival and the PDW or serum CRP level at each time point. To assess the association between PDW at each time point and survival, we divided the patients into high and low groups using the respective upper quartile values for PDW at each time point as cut-off values. Patients with low pretherapeutic PDWs ( $\leq 12.4 \mathrm{fl}, \mathrm{n}=126)$ had significantly poorer prognoses than those with high pretherapeutic PDWs $(>12.4 \mathrm{fl}, \mathrm{n}=42$ ) [5-year overall survival (OS): $60.6 \%$ vs. $75.3 \%, p=0.044$, Figure 1a]. When we similarly divided the patients into two groups using the respective upper quartile values for serum CRP levels at each time point as cut-off values, we found that patients with high postoperative serum CRP levels ( $\geq 0.5 \mathrm{mg} / \mathrm{dl}, \mathrm{n}=49$ ) showed clearly poorer survival than those with low postoperative serum CRP levels $(<0.5$ $\mathrm{mg} / \mathrm{dl}, \mathrm{n}=119$ ) (5-year OS: $33.6 \%$ vs. $71.9 \%, p<0.001$, Figure 1f). Moreover, univariate analyses for 5 -year OS revealed that low pretherapeutic PDW ( $\leq 12.4 \mathrm{fL})$ and high postoperative serum CRP levels $(\geq 0.5 \mathrm{mg} / \mathrm{dl}$ ) were significant prognostic factors [hazard ratio $(\mathrm{HR})=2.130,95 \%$ confidence interval $(\mathrm{CI})=1.062-4.880, p=0.049$ and $\mathrm{HR}=2.937,95 \% \mathrm{CI}=1.680$ $5.135, p<0.001$, respectively Figure 1a, f). On the other hand, we found no obvious differences in OS between two groups divided according to the respective cut-off values of PDW or CRP levels in other time points (Figure 1b, c, d, e).

Clinical characteristics of patients stratified according to CPC score. Based on the results summarized above, a combined PDW and CRP (CPC) score was calculated as follows: patients with both a low pretherapeutic PDW $(\leq 12.4$ fl) and high postoperative serum CRP levels $(\geq 0.5 \mathrm{mg} / \mathrm{dl})$ were assigned a score of 2 , while patients with one or neither of those were assigned a score of 1 or 0 . The clinicopathological features of the groups with each score are summarized in Table II. Regarding ypTNM staging, the score-2 group had more advanced tumor depth $(p=0.025)$ and ypStage $(p=0.017)$ than the other groups, and showed a lower rate of histological response to NAT (Grade 3) than the other groups $(p=0.002)$. The score- 2 group also had longer operation times $(p=0.010)$, longer postoperative hospital stays $(p=0.035)$, and greater intraoperative blood loss $(p=0.010)$ than the other groups. In addition, the recurrence rate and the rate of cancer death were higher in the score- 2 group than the other groups $(p=0.010$ and 0.004 , respectively). For all other prognostic markers (CAR, CONUT, LMR, NLR, PLR, PNI and mGPS), the values exhibited by the score 2 group were poorer than those exhibited by the other groups.

Overall or recurrence-free survival and the CPC score. Using Kaplan-Meier analyses, the 5-year OS for the CPC score- 0 , score- 1 , and score-2 groups were $85.1 \%, 67.7 \%$, and $26.8 \%$, respectively (pairwise multiple comparison of the Kaplan-Meier curves: the score-0 vs. score-2 group, 
Table I. Clinical characteristics of the patients $(n=168)$.

\begin{tabular}{|c|c|}
\hline & Median (IQR) or Number (\%) \\
\hline Age (years) & $64(59-68)$ \\
\hline Gender, male/female & $145(86.3) / 23(13.7)$ \\
\hline Pretherapeutic BMI $\left(\mathrm{kg} / \mathrm{m}^{2}\right)$ & $20.6(18.7-23.2)$ \\
\hline Tumor histology, AC/SCC & $6(3.6) / 162(96.4)$ \\
\hline NAT, NAC or NACRT & $21(12.5) / 147(87.5)$ \\
\hline Rate of NAT completion & $148(88.1)$ \\
\hline NAT-induced adverse events ( $\geq$ Grade 3 ) & $86(51.2)$ \\
\hline Interval between NAT and esophagectomy (days) & $37(29-46)$ \\
\hline Clinical tumor location, Upper/Middle/Lower & $30(17.9) / 81(48.2) / 57(33.9)$ \\
\hline $\mathrm{cT}, \mathrm{T} 1 / 2 / 3 / 4 \mathrm{a} / 4 \mathrm{~b}$ & $7(4.2) / 15(8.9) / 142(84.5) / 3(1.8) / 1(0.6)$ \\
\hline $\mathrm{cN}, \mathrm{N} 0 / 1 / 2 / 3$ & $25(14.9) / 96(57.1) / 46(27.3) / 1(0.6)$ \\
\hline cM (nonregional lymph node involvement ${ }^{\dagger}$ ), M0/1 & $149(88.7) / 19(11.3)$ \\
\hline cStage, I/II/III/IVA/IVB & $6(3.6) / 32(19.0) / 106(63.1) / 6(3.6) / 18(10.7)$ \\
\hline урT, $00 / 1 / 2 / 3 / 4 \mathrm{a}$ & $46(27.3) / 28(16.7) / 20(11.9) / 67(39.9) / 7(4.2)$ \\
\hline ypN, N0/1/2/3 & $95(56.5) / 39(23.2) / 28(16.7) / 6(3.6)$ \\
\hline pM (nonregional lymph node involvement $\dagger$ ), M0/1 & $154(91.7) / 14(8.3)$ \\
\hline ypStage, I/II/IIIA/IIIB/IVA/IVB & $58(34.5) / 30(17.9) / 20(11.9) / 38(22.6) / 8(4.8) / 14(8.3)$ \\
\hline Tumor differentiation, G1/G2/G3 & $31(18.5) / 111(66.1) / 26(15.5)$ \\
\hline Histological response to NAT, Grade $1 / 2 / 3$ & $64(38.1) / 70(41.7) / 34(20.2)$ \\
\hline Surgical approach, transthoracic/thoracoscopic & $121(72.0) / 47(28.0)$ \\
\hline Lymph-node dissection, 2-fields/3-fields & $7(4.2) / 161(95.8)$ \\
\hline Operation time (min) & $571(500-640)$ \\
\hline Blood loss (ml) & $553(370-756)$ \\
\hline \multicolumn{2}{|l|}{ Major complications (Clavien-Dindo $\geq$ Grade IIIa) } \\
\hline Overall & $50(29.8)$ \\
\hline Anastomotic leakage & $8(4.8)$ \\
\hline Pneumonitis & $27(16.1)$ \\
\hline Recurrent nerve paralysis & $16(9.5)$ \\
\hline Chylothorax & $10(6.0)$ \\
\hline Postoperative mortality & $2(1.2)$ \\
\hline Postoperative hospital stay (days) & $28(22-42)$ \\
\hline Recurrence rate & $63(37.5)$ \\
\hline Prognosis, alive /cancer death /death from other disease & $113(67.3) / 46(27.4) / 9(5.4)$ \\
\hline Platelet, $\left(\times 10^{4} / \mu 1\right)$ pretherapeutic/preoperative/postoperative & $24.4(20.6-29.5) / 20.4(16.4-23.9) / 20.9(17.2-25.7)$ \\
\hline lymphocyte, $(/ \mu \mathrm{l})$ pretherapeutic/preoperative/postoperative & $1610(1,330-1,947) / 965(722-1,317) / 950(770-1,270)$ \\
\hline Neutrophil, $(/ \mu \mathrm{l})$ pretherapeutic/preoperative/postoperative & $3820(2,870-5,145) / 2,335(1,735-3,275) / 2,830(2,175-3,645)$ \\
\hline Monocyte, $(/ \mu l)$ pretherapeutic/preoperative/postoperative & $380(300-460) / 380(280-480) / 325(240-427)$ \\
\hline MPV, (fL) pretherapeutic/preoperative/postoperative & $10.1(9.4-10.6) / 9.5(9.0-10.0) / 9.3(8.8-9.9)$ \\
\hline PDW, (fL) pretherapeutic/preoperative/postoperative & $11.1(10.2-12.4) / 10.2(9.1-11.2) / 9.9(8.9-10.9)$ \\
\hline Albumin, $(\mathrm{g} / \mathrm{dl})$ pretherapeutic/preoperative/postoperative & $4.0(3.8-4.2) / 3.8(3.5-4.1) / 3.9(3.6-4.2)$ \\
\hline $\mathrm{CRP},(\mathrm{mg} / \mathrm{dl})$ pretherapeutic/preoperative/postoperative & $0.13(0.06-0.51) / 0.14(0.06-0.41) / 0.13(0.05-0.46)$ \\
\hline Total cholesterol, $(\mathrm{mg} / \mathrm{dl})$ pretherapeutic/preoperative/postoperative & $185(167-212) / 201(169-224) / 185(159-213)$ \\
\hline
\end{tabular}

thateral cervical lymph node involvement. IQR: Interquartile range; BMI: body mass index; AC: adenocarcinoma; SCC: squamous cell carcinoma; NAT: neoadjuvant therapy; NAC: neoadjuvant chemotherapy; NACRT: neoadjuvant chemoradiotherapy; MPV: mean platelet volume; PDW: platelet distribution width; CRP: C-reactive protein.

$p<0.001$; score-1 vs. score-2 group, $p<0.001$; score-0 vs. score-1 group, $p=0.279$ ) (Figure 2a). On the other hand, the 5 -year recurrence-free survival (RFS) for the three groups were $74.8 \%, 59.4 \%$, and $28.1 \%$, respectively (pairwise multiple comparison of the Kaplan-Meier curves: the score0 vs. score-2 group, $p<0.001$; score- 1 vs. score-2 group, $p=0.001$; score-0 vs. score-1 group, $p=0.311$ ) (Figure 2b). The score- 0 group tended to show a better OS and RFS than the score-1 group, though the difference between these two groups was not statistically significant.

Association between the CPC score and survival. We utilized Cox's proportional hazards regression models to further analyze the association between CPC scores and survival (Table III). For $\mathrm{OS}$, the multivariable analysis showed that gender $(\mathrm{HR}=21.476$, $95 \% \mathrm{CI}=2.559-180.255, \quad p=0.005), \quad$ pretherapeutic $\mathrm{BMI}$ 
a

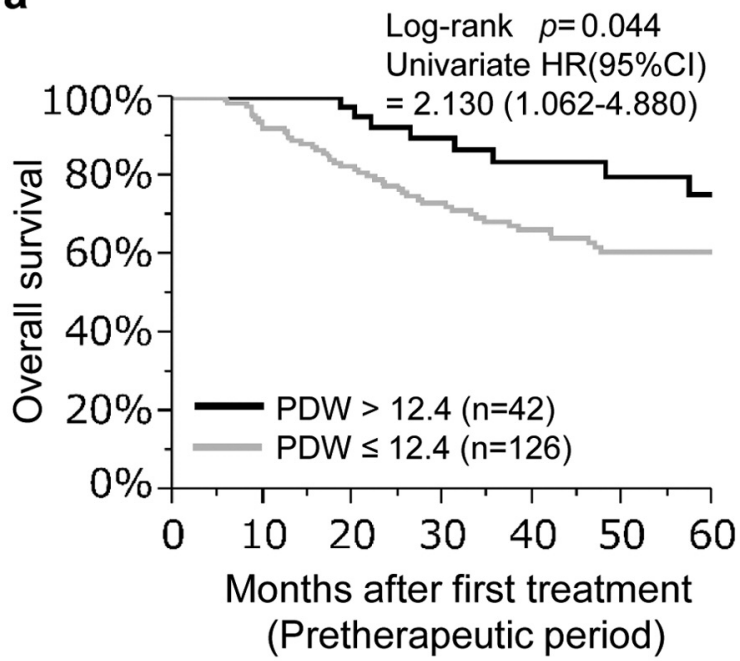

b

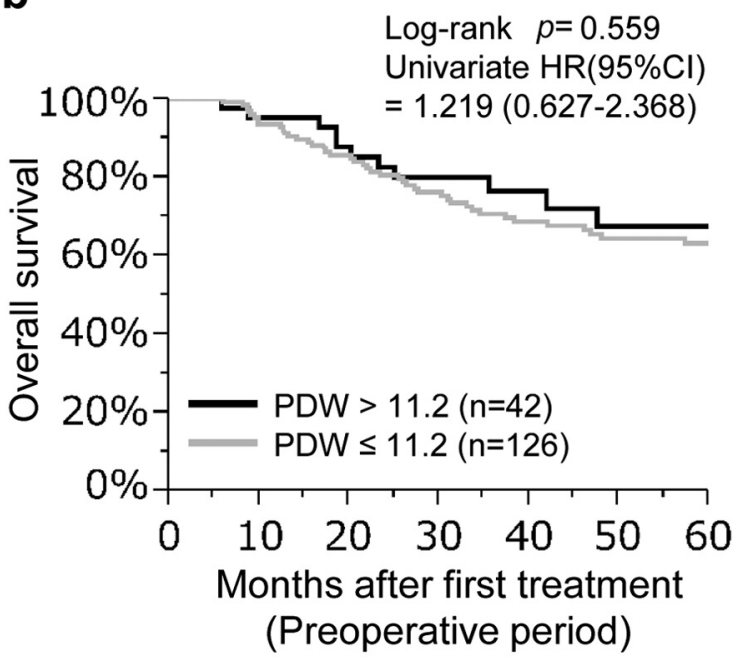

C

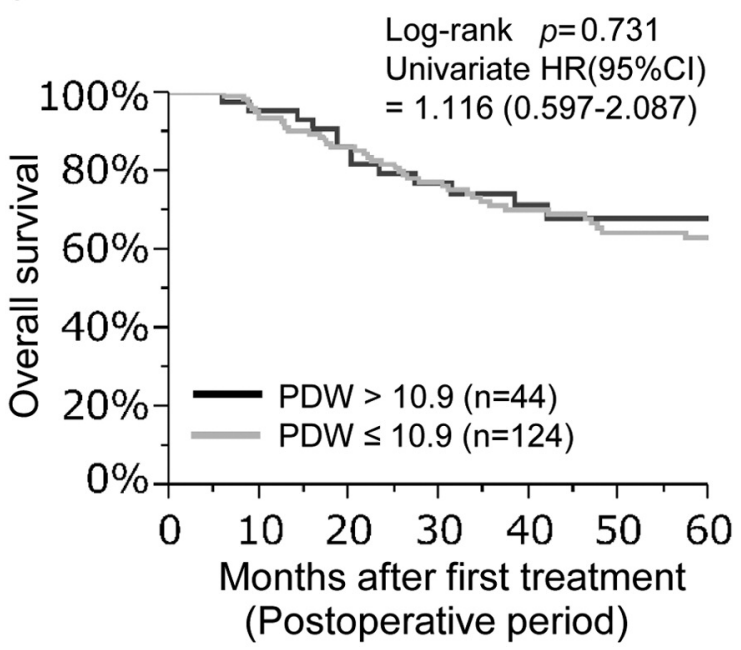

d

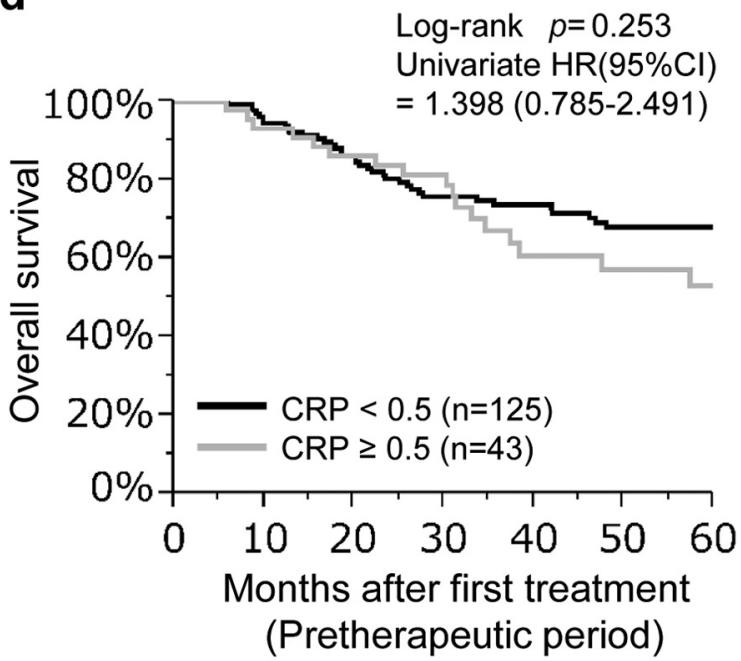

e

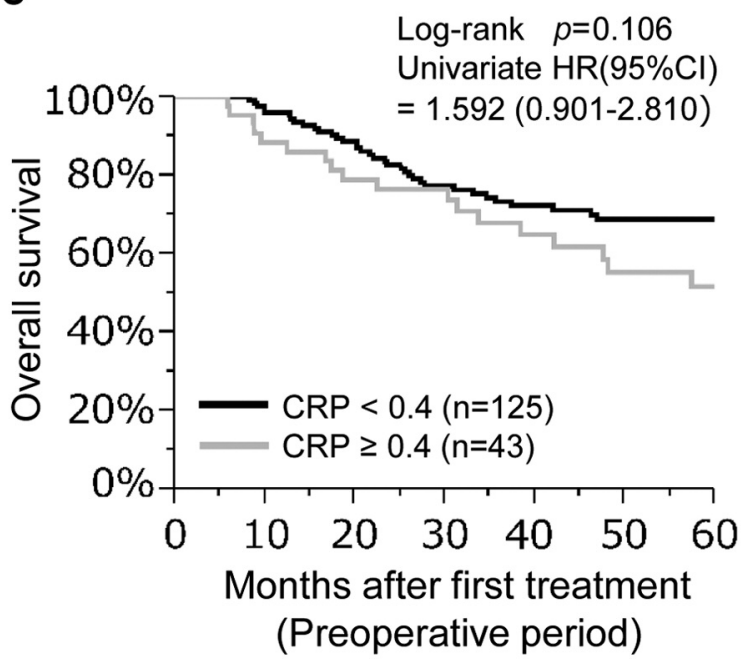

f

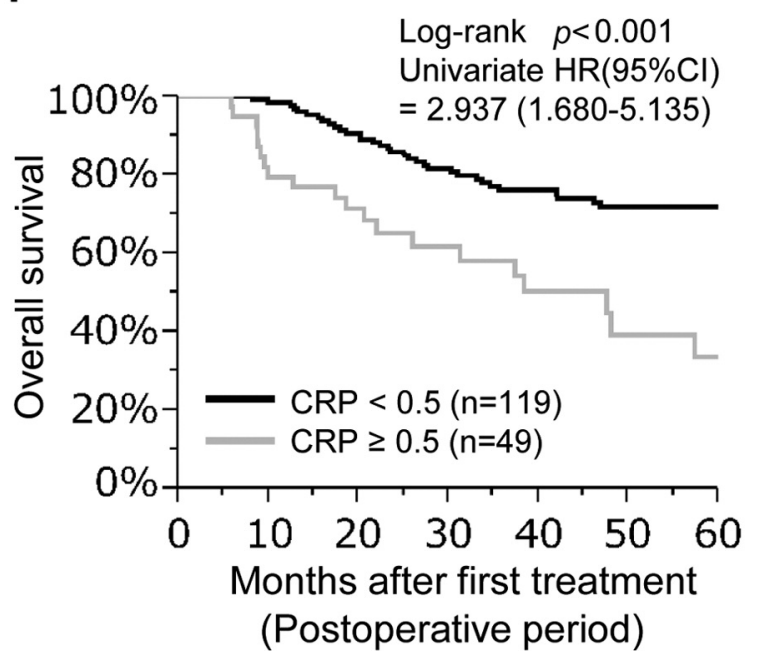

Figure 1. Kaplan-Meier and univariate Cox proportional hazards analyses of overall survival based on platelet distribution width (PDW) (a, $b, c)$ and serum C-reactive protein (CRP) levels $(d, e, f)$ at the indicated time points. HR: Hazard ratio; $95 \%$ CI: $95 \%$ confidence interval. 
Kawakita et al: CPC Score Is a Strong Prognostic Indicator of EC

Table II. Clinical characteristics of the patients after stratification according to their CPC scores.

\begin{tabular}{|c|c|c|c|c|}
\hline CPC score & $\begin{array}{l}\text { Score- } 0 \\
(\mathrm{n}=30)\end{array}$ & $\begin{array}{l}\text { Score-1 } \\
(\mathrm{n}=108)\end{array}$ & $\begin{array}{l}\text { Score-2 } \\
(\mathrm{n}=30)\end{array}$ & $p$-Value \\
\hline Age, years (range) & $65(59-69)$ & $63(58-67)$ & $64(58-68)$ & 0.563 \\
\hline Gender, male/female, $\mathrm{n}$ & $25 / 5$ & $91 / 17$ & $29 / 1$ & 0.189 \\
\hline Pretherapeutic BMI, $\mathrm{kg} / \mathrm{m}^{2}$ (range) & $21.4(19.1-24.0)$ & $20.8(18.5-23.1)$ & $19.5(18.7-23.2)$ & 0.291 \\
\hline NAT, NAC/NACRT, n & $5 / 25$ & $9 / 99$ & $7 / 23$ & 0.067 \\
\hline Rate of NAT completion, $\mathrm{n}(\%)$ & $28(93.3)$ & $94(87.0)$ & $26(86.7)$ & 0.619 \\
\hline NAT-induced adverse events ( $\geq$ Grade 3 ), n (\%) & $10(33.3)$ & $58(53.7)$ & $18(60.0)$ & 0.081 \\
\hline Interval between NAT and esophagectomy, days (range) & $37(30-45)$ & $36(28-46)$ & $43(31-48)$ & 0.397 \\
\hline Tumor location, Upper/Middle/Lower, $\mathrm{n}$ & 7/14/9 & 19/52/37 & 4/15/11 & 0.897 \\
\hline $\mathrm{cT}, \mathrm{T} 1 / 2 / 3 / 4 \mathrm{a} / 4 \mathrm{~b}, \mathrm{n}$ & $2 / 5 / 23 / 0 / 0$ & $4 / 9 / 92 / 3 / 0$ & $1 / 1 / 27 / 3 / 1$ & 0.247 \\
\hline $\mathrm{cN}, \mathrm{N} 0 / 1 / 2 / 3, \mathrm{n}$ & $8 / 13 / 9 / 0$ & $15 / 67 / 25 / 1$ & $2 / 16 / 12 / 0$ & 0.183 \\
\hline $\mathrm{cM}$ (nonregional lymph node involvement $\dagger$ ), M0/1, n & $28 / 2$ & $95 / 13$ & $26 / 4$ & 0.662 \\
\hline cStage I-II/III-IV, n & $10 / 20$ & $24 / 84$ & $3 / 27$ & 0.092 \\
\hline урT, T0/1/2/3/4a, n & $7 / 9 / 2 / 11 / 1$ & $36 / 17 / 14 / 38 / 3$ & $3 / 2 / 4 / 18 / 3$ & 0.025 \\
\hline $\mathrm{ypN}, \mathrm{N} 0 / 1 / 2 / 3, \mathrm{n}$ & $22 / 7 / 1 / 0$ & $62 / 22 / 20 / 4$ & $11 / 10 / 7 / 2$ & 0.077 \\
\hline pM (nonregional lymph node involvement ${ }^{\dagger}$ ), M0/1, n & $30 / 0$ & $96 / 12$ & $28 / 2$ & 0.140 \\
\hline ypStage I-II/III-IV, n & $21 / 9$ & $57 / 51$ & $10 / 20$ & 0.017 \\
\hline Tumor differentiation, G1/G2/G3, n & $7 / 17 / 6$ & 20/72/16 & $4 / 22 / 4$ & 0.740 \\
\hline Histological response to NAT, Grade $1 / 2 / 3, \mathrm{n}$ & 9/14/7 & $34 / 48 / 26$ & 21/8/1 & 0.002 \\
\hline Surgical approach, transthoracic/thoracoscopic, $\mathrm{n}$ & $21 / 9$ & $79 / 29$ & $21 / 9$ & 0.190 \\
\hline Operation time, min (range) & $562(516-625)$ & $563(478-619)$ & 637 (554-699) & 0.010 \\
\hline Intraoperative blood loss, ml (range) & $556(301-789)$ & $494(331-707)$ & $688(541-812)$ & 0.010 \\
\hline Complications (Clavien-Dindo $\geq$ Grade IIIa), n (\%) & $6(20.0)$ & $31(28.7)$ & $13(43.3)$ & 0.131 \\
\hline Postoperative hospital stay, days (range) & $28(24-38)$ & $26(21-41)$ & $31(25-73)$ & 0.035 \\
\hline Recurrence rate, n (\%) & $7(23.3)$ & $38(35.2)$ & $18(60.0)$ & 0.010 \\
\hline Prognosis, alive/cancer death /death from other disease, $\mathrm{n}$ & $25 / 4 / 1$ & $76 / 28 / 4$ & $12 / 14 / 4$ & 0.004 \\
\hline Pretherapeutic CAR (range) & $0.028(0.012-0.079)$ & $0.032(0.013-0.134)$ & $0.059(0.018-0.169)$ & 0.536 \\
\hline Preoperative CAR (range) & $0.044(0.016-0.191)$ & $0.035(0.015-0.085)$ & $0.041(0.020-0.278)$ & 0.407 \\
\hline Postoperative CAR (range) & $0.015(0.010-0.030)$ & $0.026(0.011-0.067)$ & $0.331(0.225-0.982)$ & $<0.001$ \\
\hline Pretherapeutic CONUT score (range) & $1(0-2)$ & $1(0-2)$ & $1(1-3)$ & 0.338 \\
\hline Preoperative CONUT score (range) & $2(1-3)$ & $3(2-4)$ & $4(2-5)$ & 0.048 \\
\hline Postoperative CONUT score (range) & $2(2-3)$ & $2(1-3)$ & $3(2-6)$ & 0.002 \\
\hline Pretherapeutic LMR (range) & $4.32(3.26-5.89)$ & $4.39(3.23-5.80)$ & $4.04(3.24-5.44)$ & 0.838 \\
\hline Preoperative LMR (range) & $2.61(1.86-4.17)$ & $2.75(1.93-3.67)$ & $2.39(1.92-3.49)$ & 0.906 \\
\hline Postoperative LMR (range) & $3.38(2.24-4.88)$ & $3.00(2.41-4.71)$ & $2.06(1.41-3.81)$ & 0.006 \\
\hline Pretherapeutic NLR (range) & $2.30(1.69-2.72)$ & $2.40(1.79-3.39)$ & $2.48(1.67-3.06)$ & 0.540 \\
\hline Preoperative NLR (range) & $2.28(1.80-3.92)$ & $2.56(1.85-3.60)$ & $3.04(1.81-5.14)$ & 0.570 \\
\hline Postoperative NLR (range) & $2.76(1.67-3.37)$ & $2.65(1.99-3.93)$ & $3.62(3.08-8.12)$ & 0.009 \\
\hline Pretherapeutic PLR (range) & $132(100-168)$ & $168(127-198)$ & $169(129-205)$ & 0.008 \\
\hline Preoperative PLR (range) & $184(120-281)$ & $214(169-270)$ & $225(161-357)$ & 0.157 \\
\hline Postoperative PLR (range) & $174(137-211)$ & $208(158-294)$ & $322(212-409)$ & $<0.001$ \\
\hline Pretherapeutic PNI (range) & $48.1(45.3-52.3)$ & $48.4(44.6-52.6)$ & $46.8(43.9-50.2)$ & 0.641 \\
\hline Preoperative PNI (range) & $43.4(39.8-46.2)$ & $43.5(40.1-46.1)$ & $42.4(38.4-45.5)$ & 0.593 \\
\hline Postoperative PNI (range) & $44.8(41.3-48.3)$ & $44.7(41.5-48.1)$ & $40.1(35.3-43.8)$ & $<0.001$ \\
\hline Pretherapeutic mGPS, Score $0 / 1 / 2, n$ & 28/1/1 & 92/9/7 & $24 / 5 / 1$ & 0.387 \\
\hline Preoperative mGPS, Score $0 / 1 / 2, n$ & $20 / 8 / 2$ & $80 / 23 / 5$ & $18 / 8 / 4$ & 0.416 \\
\hline Postoperative mGPS, Score $0 / 1 / 2, n$ & $25 / 5 / 0$ & $93 / 12 / 3$ & $8 / 13 / 9$ & $<0.001$ \\
\hline
\end{tabular}

Statistically significant values $(p<0.05)$ are given in bold. ${ }^{\dagger}$ Lateral cervical lymph node involvement. CPC Score: Combined platelet distribution width and C-reactive protein score; BMI: body mass index; NAT: neoadjuvant therapy; NAC: neoadjuvant chemotherapy; NACRT: neoadjuvant chemoradiotherapy; CAR: C-reactive protein-to-albumin ratio; CONUT score: controlling nutritional status score; LMR lymphocyte-to-monocyte ratio; NLR: neutrophil-to-lymphocyte ratio; PLR: platelet-to-lymphocyte ratio; PNI: prognostic nutritional index; mGPS: modified Glasgow prognostic score.

$(\mathrm{HR}=0.811,95 \% \mathrm{CI}=0.715-0.917, p<0.001), \mathrm{ypN}(\mathrm{HR}=2.066$, $95 \% \mathrm{CI}=1.388-3.105, p<0.001), \quad \mathrm{CPC}$ score $(\mathrm{HR}=2.639$, 95\% CI $=1.327-5.322, p=0.006)$, and postoperative NLR $(\mathrm{HR}=1.154,95 \% 1.037-1.369, p=0.002)$ were all independent factors contributing to OS. In addition, the multivariable analysis revealed that $\mathrm{ypN}(\mathrm{HR}=1.539,95 \% \mathrm{CI}=1.174-2.018$, $p=0.002)$, ypM $(\mathrm{HR}=2.370,95 \% \mathrm{CI}=1.088-5.166, p=0.030)$, the CPC score $(\mathrm{HR}=1.890,95 \% \mathrm{CI}=1.217-2.936, p=0.004)$, and 
a

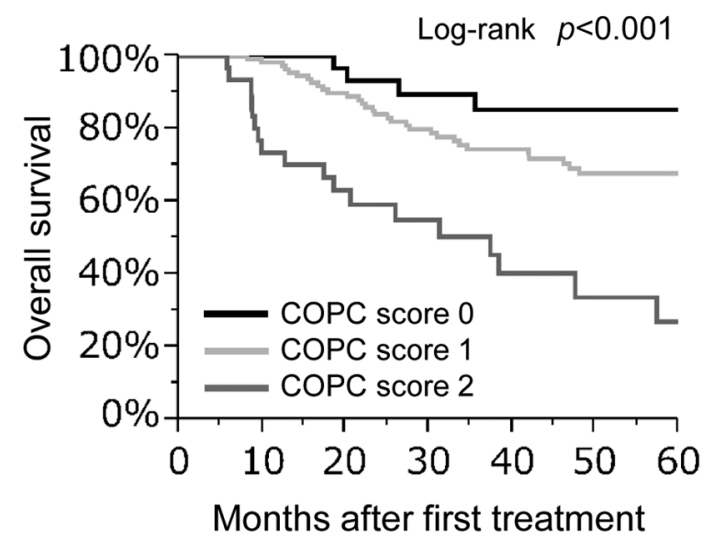

Number at risk

$\begin{array}{lccccccc}\text { Score 0 } & 30 & 30 & 29 & 23 & 20 & 18 & 13 \\ \text { Score 1 } & 108 & 107 & 94 & 76 & 61 & 50 & 41 \\ \text { Score 2 } & 30 & 23 & 17 & 13 & 8 & 6 & 4\end{array}$

b

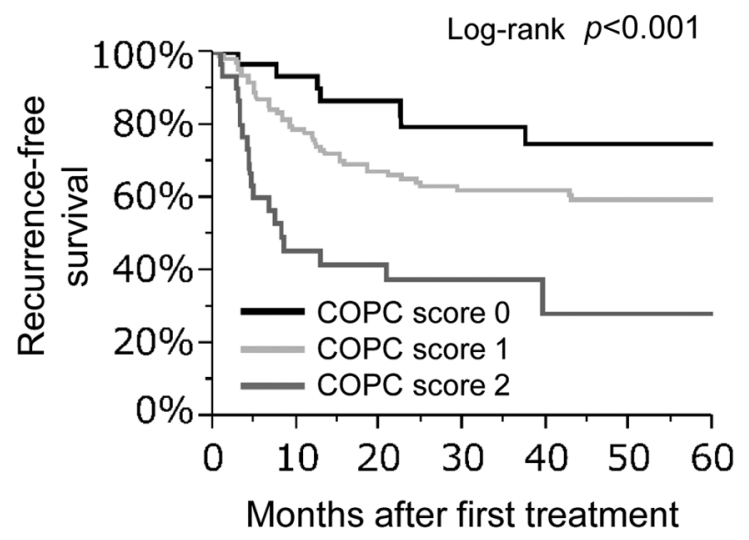

Number at risk

$\begin{array}{llllllll}\text { Score 0 } & 30 & 29 & 25 & 20 & 16 & 14 & 10 \\ \text { Score 1 } & 108 & 86 & 68 & 56 & 49 & 42 & 33 \\ \text { Score 2 } & 30 & 13 & 11 & 7 & 4 & 2 & 1\end{array}$

Figure 2. Overall (a) and recurrence-free survival (b) stratified according to the CPC score. CPC score is an abbreviation for a combined platelet distribution width $(P D W)$ and C-reactive protein $(C R P)$ score and was defined as follows: patients with both low pretherapeutic PDW ( $\leq 12.4 \mathrm{fl})$ and high postoperative serum CRP levels $(\geq 0.5 \mathrm{mg} / \mathrm{dl})$ were assigned a score of 2 , while patients with one or neither of those were assigned a score of 1 and 0 .

preoperative $\mathrm{mGPS}(\mathrm{HR}=1.506,95 \% \mathrm{CI}=1.032-2.198, p=0.042)$ were independent factors contributing to RFS.

Subgroup analysis stratified according to clinicopathological features. Kaplan-Meier subgroup analyses revealed that CPC scores correlated significantly with OS, regardless of whether patients were ypN-negative or -positive or ypStage I-II or III-VI $(\log$ rank $p=0.012,0.002,0.007$, and 0.004 , respectively) (Figure 3). Moreover, the differences in OS between the score- 0 and score- 1 groups tended to be more remarkable among the patients with more advanced disease (node-positive or ypStage III-IV). The univariate analyses demonstrated that the CPC score was clearly related to OS, irrespective of age, tumor location, NAT, the presence or absence lymph node involvement, ypT or ypTNM Stage (Table IV).

\section{Discussion}

In this article, we showed for the first time that the combined pretherapeutic PDW and postoperative serum CRP levels produces a stronger predictor of prognosis in EC patients than other known prognostic markers. Although the CPC score reflected the ypTNM Stage of EC patients to some extent, the score-0 group included patients at more advanced Stages (ypStage III; 30\% in the score-0 group), while the score-2 group contained patients at less advanced Stages (ypStage I-II; total $33.3 \%$ in the score-2 group). Consequently, the advantage of the CPC score is that it enables detection of EC patients with favorable and poor prognoses, irrespective of ypTNM Stage.

Most studies of the association between PDW and malignancies have shown that high PDWs correlate significantly with poor prognoses (8-12). Within the tumor microenvironment, tumor cells induce platelet activation and aggregation, and enhance thrombopoiesis by releasing thrombopoietic cytokines, such as interleukin-6 (IL-6) and tumor-derived platelet factor 4 (PF4) (7). The activated platelets then release granules containing numerous tumor promoting factors, including platelet-derived growth factor (PDGF), vascular endothelial growth factor (VEGF) and transforming growth factor $\beta$ (TGF- $\beta$ ), which is followed by the release of extracellular vesicles (EVs) such as platelet-derived microparticles (PMPs) $(7,28)$. These various mediators then collectively act to facilitate tumor cell growth and proliferation, angiogenesis, and epithelial mesenchymal transition (EMT) $(7,28)$. The PDW is reported to more sensitively reflect variation in platelet volume related to pseudopodia formation and shape changes during platelet activation than MPV; consequently, elevated PDW is considered to be an indicator of platelet activation (6). 
Kawakita et al: CPC Score Is a Strong Prognostic Indicator of EC

Table III. Univariate and multivariable analyses of survival (Cox's proportional hazards regression models).

\begin{tabular}{|c|c|c|c|c|c|c|}
\hline \multirow[b]{3}{*}{ Factor } & \multicolumn{3}{|c|}{ OS } & \multicolumn{3}{|c|}{ RFS } \\
\hline & \multirow{2}{*}{$\begin{array}{l}\text { Univariate } \\
p \text {-Value }\end{array}$} & \multicolumn{2}{|c|}{ Multivariable } & \multirow{2}{*}{$\begin{array}{c}\text { Univariate } \\
p \text {-Value }\end{array}$} & \multicolumn{2}{|c|}{ Multivariable } \\
\hline & & Hazard ratio $(95 \% \mathrm{CI})$ & $p$-Value & & Hazard ratio $(95 \% \mathrm{CI})$ & $p$-Value \\
\hline Age (years)* & 0.796 & - & - & 0.168 & - & - \\
\hline Gender (male vs. female) & $<0.001$ & $21.476(2.559-180.255)$ & 0.005 & 0.235 & - & - \\
\hline Pretherapeutic BMI* & 0.010 & $0.811(0.715-0.917)$ & $<0.001$ & 0.120 & - & - \\
\hline $\mathrm{cT}(1,2,3,4)^{*}$ & 0.063 & - & - & 0.025 & $1.186(0.719-1.955)$ & 0.504 \\
\hline $\mathrm{cN}(1,2,3)^{*}$ & 0.077 & - & - & 0.179 & - & - \\
\hline \multicolumn{7}{|l|}{$\mathrm{cM}^{-n o n r e g i o n a l}$ lymph node involvement ${ }^{\dagger}$} \\
\hline (Yes vs. No) & 0.247 & - & - & 0.181 & $1.186(0.719-1.955)$ & - \\
\hline урТ $(0,1,2,3,4)^{*}$ & 0.003 & $1.027(0.724-1.463)$ & 0.882 & $<0.001$ & $1.326(0.969-1.814)$ & 0.077 \\
\hline $\mathrm{ypN}(0,1,2,3)^{*}$ & $<0.001$ & $2.066(1.388-3.105)$ & $<0.001$ & $<0.001$ & $1.539(1.174-2.018)$ & 0.002 \\
\hline \multicolumn{7}{|l|}{ ypM-nonregional lymph node involvement ${ }^{\dagger}$} \\
\hline Tumor histology (SCC vs. AC) & 0.856 & - & - & 0.724 & - & - \\
\hline Tumor differentiation $(\mathrm{G} 1,2,3)^{*}$ & 0.992 & - & - & 0.717 & - & - \\
\hline NAT (NACRT $v s$. NAC) & 0.530 & - & - & 0.522 & - & - \\
\hline \multicolumn{7}{|l|}{ Interval between NAT and surgery } \\
\hline ( $\geq 37$ days $v s . \leq 36$ days) & 0.629 & - & - & 0.172 & - & - \\
\hline \multicolumn{7}{|l|}{ NAT-induced adverse events } \\
\hline Histological response to NAT (Grade 1, 2, 3)* & $<0.001$ & $0.935(0.460-1.878)$ & 0.851 & $<0.001$ & $1.020(0.591-1.759)$ & 0.943 \\
\hline \multicolumn{7}{|l|}{ Surgical approach } \\
\hline (transthoracic $v s$. thoracoscopic) & 0.988 & - & - & 0.683 & - & - \\
\hline Lymph node dissection (2-fields $v s .3$-fields) & 0.550 & - & - & 0.552 & - & - \\
\hline \multicolumn{7}{|l|}{ Mojor Complication (Clavien-Dindo $\geq \mathrm{IIIa}$ ) } \\
\hline (Yes vs. No) & 0.031 & $1.696(0.865-3.326)$ & 0.124 & 0.159 & - & - \\
\hline Perioperative blood transfusion (Yes $v s$. No) & 0.184 & - & - & 0.492 & - & - \\
\hline CPC score* & $<0.001$ & $2.639(1.327-5.322)$ & 0.006 & $<0.001$ & $1.890(1.217-2.936)$ & 0.004 \\
\hline Pretherapeutic MPV* & 0.029 & $1.152(0.722-1.846)$ & 0.554 & 0.231 & - & - \\
\hline Pretherapeutic CAR* & 0.032 & $1.771(0.663-4.198)$ & 0.237 & 0.200 & - & - \\
\hline Pretherapeutic CONUT* & 0.360 & - & - & 0.420 & - & - \\
\hline Pretherapeutic LMR* & 0.844 & - & - & 0.531 & - & - \\
\hline Pretherapeutic NLR* & 0.467 & - & - & 0.469 & - & - \\
\hline Pretherapeutic PLR* & 0.099 & - & - & 0.368 & - & - \\
\hline Pretherapeutic PNI* & 0.120 & - & - & 0.793 & - & - \\
\hline Pretherapeutic mGPS* & 0.083 & - & - & 0.571 & - & - \\
\hline Preoperative MPV* & 0.361 & - & - & 0.434 & - & - \\
\hline Preoperative CAR* & 0.234 & - & - & 0.056 & - & - \\
\hline Preoperative CONUT* & 0.268 & - & - & 0.270 & - & - \\
\hline Preoperative LMR* & 0.420 & - & - & 0.391 & - & - \\
\hline Preoperative NLR* & 0.141 & - & - & 0.509 & - & - \\
\hline Preoperative PLR* & 0.067 & - & - & 0.343 & - & - \\
\hline Preoperative PNI* & 0.451 & - & - & 0.497 & - & - \\
\hline Preoperative mGPS* & 0.015 & $1.003(0.564-1.699)$ & 0.993 & 0.021 & $1.506(1.032-2.198)$ & 0.042 \\
\hline Postoperative MPV* & 0.301 & - & - & 0.945 & - & - \\
\hline Postoperative CAR* & 0.005 & $0.872(0.315-2.141)$ & 0.788 & 0.270 & - & - \\
\hline Postoperative CONUT* & 0.174 & - & - & 0.644 & - & - \\
\hline Postoperative LMR* & 0.319 & - & - & 0.999 & - & - \\
\hline Postoperative NLR* & $<0.001$ & $1.154(1.037-1.369)$ & 0.002 & 0.107 & - & - \\
\hline Postoperative PLR* & 0.647 & - & - & 0.991 & - & - \\
\hline Postoperative PNI* & 0.127 & - & - & 0.825 & - & - \\
\hline Postoperative mGPS* & 0.018 & $0.529(0.241-1.072)$ & 0.079 & 0.098 & - & - \\
\hline
\end{tabular}

Statistically significant values $(p<0.05)$ are given in bold. $*$ The hazard ratio for a one unit change; ${ }^{\dagger}$ Lateral cervical lymph node involvement. OS: Overall survival; RFS: recurrence-free survival; BMI: body mass index; AC: adenocarcinoma; SCC: squamous cell carcinoma; NAT: neoadjuvant therapy; NAC: neoadjuvant chemotherapy; NACRT: neoadjuvant chemoradiotherapy; CPC score: combined platelet distribution width and C-reactive protein score; MPV: mean platelet volume; CAR: C-reactive protein-to-albumin ratio; CONUT score: controlling nutritional status score; LMR: lymphocyte-to-monocyte ratio; NLR: neutrophil-to-lymphocyte ratio; PLR: platelet-to-lymphocyte ratio; PNI: prognostic nutritional index; mGPS: modified Glasgow prognostic score. 
a

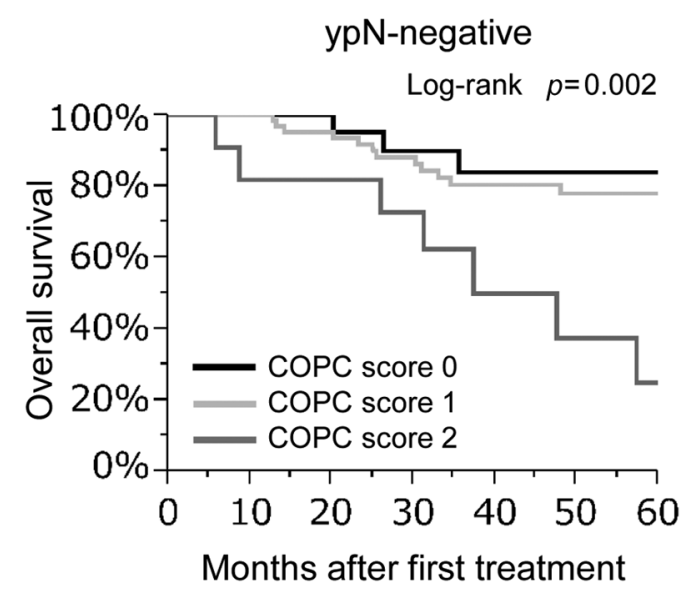

Number at risk

$\begin{array}{llllllll}\text { Score 0 } & 22 & 22 & 22 & 17 & 14 & 13 & 10 \\ \text { Score 1 } & 63 & 63 & 57 & 47 & 38 & 33 & 25 \\ \text { Score 2 } & 11 & 10 & 10 & 8 & 5 & 4 & 2\end{array}$

C

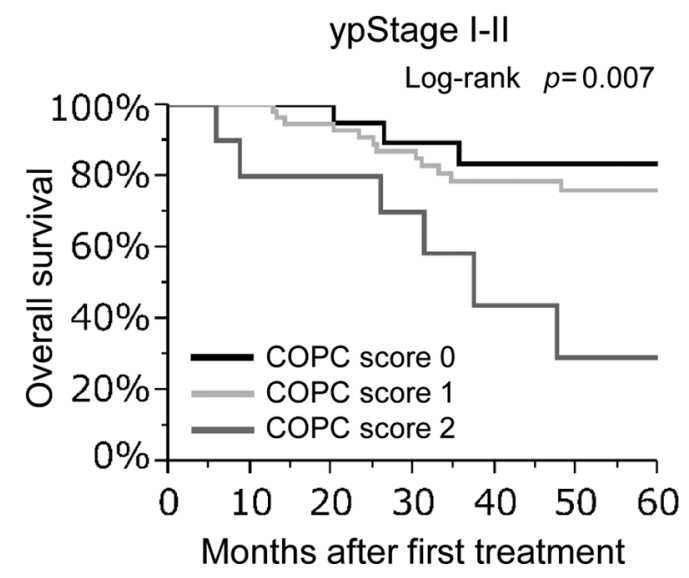

Number at risk

$\begin{array}{llllllll}\text { Score 0 } & 21 & 21 & 21 & 16 & 14 & 13 & 10 \\ \text { Score 1 } & 57 & 57 & 52 & 43 & 35 & 30 & 23 \\ \text { Score 2 } & 10 & 10 & 9 & 7 & 4 & 3 & 2\end{array}$

b

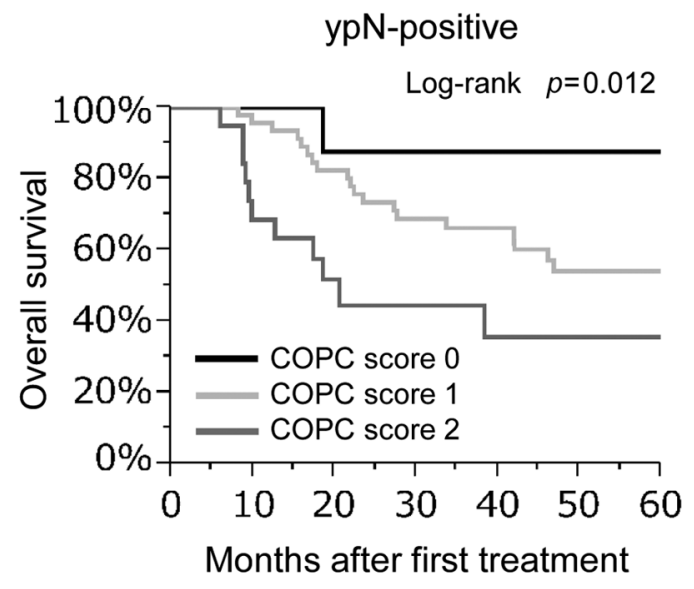

d

$\begin{array}{cccccccr}\text { Score 0 } & 8 & 8 & 8 & 7 & 7 & 6 & 3 \\ \text { Score 1 } & 45 & 44 & 38 & 30 & 24 & 18 & 16 \\ \text { Score 2 } & 19 & 14 & 8 & 6 & 4 & 3 & 2\end{array}$

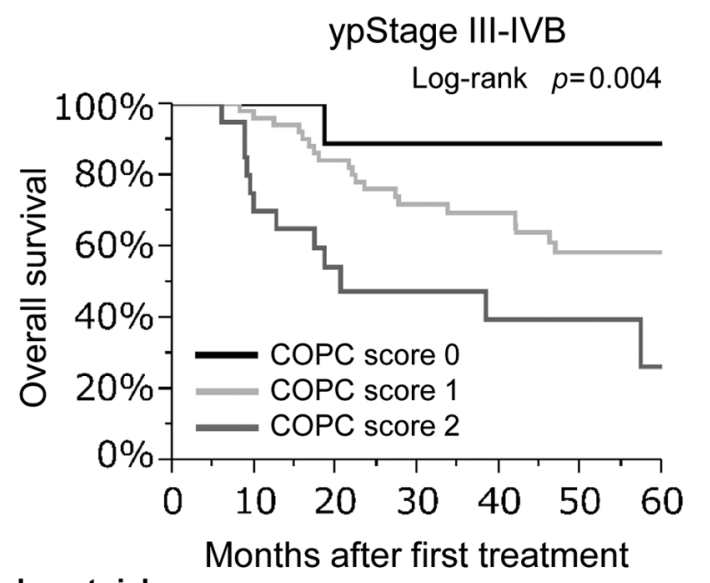

$\begin{array}{cccccccc}\text { Number at risk } & & & & \\ \text { Score 0 } & 9 & 9 & 9 & 8 & 7 & 6 & 3 \\ \text { Score 1 } & 51 & 50 & 43 & 34 & 27 & 21 & 18 \\ \text { Score 2 } & 20 & 15 & 9 & 7 & 5 & 4 & 2\end{array}$

Figure 3. Subgroup analyses of overall survival based on CPC score stratified according to ypN negative or -positive and ypStage: ypN-negative (a), ypN-positive (b), ypStage I-II (c), and ypStage III-IV (d). CPC score is an abbreviation for a combined platelet distribution width (PDW) and $C$ reactive protein $(C R P)$ score and was defined as follows: patients with both low pretherapeutic PDW $(\leq 12.4$ fl) and high postoperative serum CRP levels $(\geq 0.5 \mathrm{mg} / \mathrm{dl})$ were assigned a score of 2 , while patients with one or neither of those were assigned a score of 1 and 0 .

By contrast, our findings indicate that low PDWs are related to poor outcomes, which is consistent with several earlier studies (13-16). Low MPV is reportedly predictive of unfavorable outcomes in diffuse large B-cell lymphoma (29), multiple myeloma (30), and lung cancer (31). Moreover, in an analysis of 1544 patients with various types of cancer, Riedl et al. found that patients with high MPV levels exhibited both a lower risk of venous thromboembolism and better survival than those with lower MPV levels (32). These findings suggest that smaller platelets may have a stronger 
Table IV. Subgroup univariate analyses of overall survival based on the CPC score (Cox's proportional hazards regression models).

\begin{tabular}{|c|c|c|c|}
\hline Factor & $\mathrm{N}$ & $\begin{array}{l}\text { Hazard ratio } \\
(95 \% \mathrm{CI})\end{array}$ & $p$-Value \\
\hline \multicolumn{4}{|l|}{ Age } \\
\hline$\leq 64$ & 88 & $3.613(1.860-7.092)$ & $<0.001$ \\
\hline$\geq 65$ & 80 & $2.410(1.210-4.811)$ & 0.012 \\
\hline \multicolumn{4}{|l|}{ Gender } \\
\hline Male & 145 & $2.695(1.682-4.339)$ & $<0.001$ \\
\hline Female & 23 & $2.236(0.039-129.529)$ & 0.711 \\
\hline \multicolumn{4}{|l|}{ Tumor location } \\
\hline Upper & 29 & $4.469(1.159-17.759)$ & 0.030 \\
\hline Middle or Lower & 139 & $2.727(1.636-4.560)$ & $<0.001$ \\
\hline \multicolumn{4}{|l|}{ Tumor differentiation } \\
\hline G1-2 & 142 & $3.428(2.056-5.740)$ & $<0.001$ \\
\hline G3 & 26 & $1.223(0.270-4.663)$ & 0.873 \\
\hline \multicolumn{4}{|l|}{ NAT } \\
\hline NAC & 21 & $3.617(1.211-15.408)$ & 0.020 \\
\hline NACRT & 147 & $2.840(1.645-4.889)$ & $<0.001$ \\
\hline \multicolumn{4}{|c|}{ Histological response to NAT } \\
\hline Grade 1-2 & 134 & $2.660(1.646-4.336)$ & $<0.001$ \\
\hline Grade 3 & 34 & $2.586(0.324-21.731)$ & 0.396 \\
\hline \multicolumn{4}{|l|}{ Tumor depth (T) } \\
\hline cT1-2 & 23 & $5.154(0.733-44.881)$ & 0.100 \\
\hline cT3-4 & 145 & $2.741(1.668-4.514)$ & $<0.001$ \\
\hline урТ0-2 & 88 & $2.618(1.223-5.608)$ & 0.013 \\
\hline урТ3-4 & 80 & $2.777(1.483-5.244)$ & 0.001 \\
\hline \multicolumn{4}{|c|}{ Lymph node involvement $(\mathrm{N})$} \\
\hline $\mathrm{cNO}$ & 25 & $1.197 \times 10^{9}(2.355--)$ & 0.010 \\
\hline $\mathrm{cN}+$ & 143 & $2.621(1.594-4.318)$ & $<0.001$ \\
\hline ypN0 & 96 & $2.814(1.338-5.927)$ & 0.006 \\
\hline ypN+ & 72 & $2.562(1.357-4.879)$ & 0.004 \\
\hline \multicolumn{4}{|c|}{$\begin{array}{l}\text { Nonregional lymph } \\
\text { node involvement }{ }^{\dagger}(\mathrm{M})\end{array}$} \\
\hline $\mathrm{cM} 0$ & 149 & $2.805(1.674-4.713)$ & $<0.001$ \\
\hline cM1 & 19 & $3.431(0.930-13.152)$ & 0.064 \\
\hline урм0 & 154 & $3.074(1.854-5.116)$ & $<0.001$ \\
\hline ypM1 & 14 & $2.469(0.121-19.648)$ & 0.473 \\
\hline \multicolumn{4}{|l|}{ TNM stage } \\
\hline cStage I-II & 37 & $6.431(0.913-43.834)$ & 0.062 \\
\hline cStage III-IV & 131 & $2.556(1.556-4.213)$ & $<0.001$ \\
\hline ypStage I-II & 88 & $2.618(1.223-5.608)$ & 0.013 \\
\hline ypStage III-IV & 80 & $2.778(1.483-5.244)$ & 0.001 \\
\hline
\end{tabular}

Statistically significant values $(p<0.05)$ are given in bold. †Lateral cervical lymph node involvement. CPC score: combined platelet distribution width and C-reactive protein score; OS: overall survival; NAT: neoadjuvant therapy; NAC: neoadjuvant chemotherapy; NACRT: neoadjuvant chemoradiotherapy; TNM: tumor-node-metastasis.

prothrombotic tendency and may be more supportive of tumor development than larger platelets. Platelets with a low MPV also generally exhibit a low PDW. In our study, the patients with low PDWs, had MPVs nearly as low as the "low MPVs" defined in the studies mentioned above. In the present study, however, multivariable analysis showed no significant relation between MPV and survival, though a univariate analysis revealed that pretherapeutic MPV was a significant indicator of OS. The role of platelets with a low PDW in tumor pathogenesis remains to be elucidated.

The PDW reportedly correlates with several inflammatory parameters (10). In the present study, however, the PDW only very weakly correlated with serum CRP levels at the pretherapeutic time point (data not shown). On the other hand, a low pretherapeutic PDW was a significant risk factor for $\mathrm{cN}$ - and $\mathrm{ypN}$-positivity in the multiple logistic regression analysis [odds ratio=2.681 $(p=0.035)$ and $2.458(p=0.029)$, respectively]. These findings suggest that pretherapeutic PDW may reflect the magnitude of platelet-mediated cancer promotion in the tumor microenvironment that is not directly reflected as systemic inflammation.

The present study revealed that the pretherapeutic PDW is associated with survival, though neither the preoperative nor postoperative PDW reflected survival, which is consistent with several past studies $(9,12-16)$. One possible explanation for these results is that preoperative and postoperative PDWs are not affected by tumor progression alone, but also by other factors such as bone marrow suppression due to NAT and postoperative malnutrition arising from reduced oral intake of nutrition.

It is well known that chronic inflammation plays a crucial role in the development and progression of malignant tumors and that CRP is an important indicator of inflammation $(17,33-34)$. In the present study, the univariate analyses at the three time points showed that only serum CRP levels at the postoperative time point were positively associated with OS. One possible explanation for why postoperative serum CRP levels may be particularly important is that they directly reflect the magnitude of the chronic inflammation caused by the various stresses encountered by the patient during the course of therapy, especially those related to the surgical intervention and postoperative complications.

Lastly, there are two limitations to the present study. First, because of the small number of patients, we were unable to adequately assess the effect of the CPC score on survival among patients with adenocarcinoma and female patients. Second, due to the retrospective nature of this study, we could not fully verify the role of platelets with a low PDW in tumorigenesis. We will address these issues in the next study. Nonetheless, by combining pretherapeutic PDW and postoperative serum CRP levels, we have established a novel prognostic score that is predictive of outcomes in EC patients. This CPC score could potentially help clinicians assess EC patient prognosis, irrespective of ypTNM Stage.

\section{Conflicts of Interest}

None of the Authors have any conflicts of interest to declare regarding this study. 


\section{Authors' Contributions}

Guarantors of integrity of entire study and Study concepts: Kawakita Y; Study design or data acquisition or data analysis: Motoyama S, Sato Y, Wakita A, Nagaki Y, Imai K; Statistical analysis, and manuscript editing, Kawakita Y, Motoyama S, Minamiya Y.

\section{Acknowledgements}

This work was supported, in part, by Grants-in-Aid for Scientific Research from the Ministry of Education, Culture, Science, Sports and the Japan Science and Technology Agency.

\section{References}

1 Gertler R, Stein HJ, Langer R, Nettelmann M, Schuster T, Hoefler H, Siewert JR and Feith M: Long-term outcome of 2920 patients with cancers of the esophagus and esophagogastric junction: evaluation of the New Union Internationale Contre le Cancer/American Joint Cancer Committee staging system. Ann Surg 253: 689-698, 2011. PMID: 21475008. DOI: 10.1097/ SLA.0b013e31821111b5

2 Schandl A, Kauppila JH, Anandavadivelan P, Johar A and Lagergren P: Predicting the risk of weight loss after esophageal cancer surgery. Ann Surg Oncol 26: 2385-2391, 2019. PMID: 31004297. DOI: $10.1245 / \mathrm{s} 10434-019-07352-5$

3 Shichinohe T, Uemura S, Hirano S and Hosokawa M: Impact of preoperative skeletal muscle mass and nutritional status on shortand long-term outcomes after esophagectomy for esophageal cancer: a retrospective observational study: impact of psoas muscle mass and body mass on esophagectomy. Ann Surg Oncol 26: 1301-1310, 2019. PMID: 30725311. DOI: 10.1245/s10434019-07188-z.

4 Kosumi K, Baba Y, Ishimoto T, Harada K, Nakamura K, Ohuchi M, Kiyozumi Y, Izumi D, Tokunaga R, Taki K, Higashi T, Miyata T, Kurashige J, Hiyoshi Y, Iwagami S, Sakamoto Y, Miyamoto Y, Yoshida N, Watanabe $M$ and Baba $\mathrm{H}$ : Neutrophil/lymphocyte ratio predicts the prognosis in esophageal squamous cell carcinoma patients. Surg Today 46: 405-413, 2016. PMID: 26036223. DOI: 10.1007/s00595-015-1197-0

5 Osselaer JC, Jamart J and Scheiff JM: Platelet distribution width for differential diagnosis of thrombocytosis. Clin Chem 43: 1072-1076, 1997. PMID: 9191563.

6 Vagdatli E, Gounari E, Lazaridou E, Katsibourlia E, Tsikopoulou F and Labrianou I: Platelet distribution width: a simple, practical and specific marker of activation of coagulation. Hippokratia 14 : 28-32, 2010. PMID: 20411056.

$7 \mathrm{Xu}$ XR, Yousef GM and Ni H: Cancer and platelet crosstalk: opportunities and challenges for aspirin and other antiplatelet agents. Blood 131: 1777-1789, 2018. PMID: 29519806. DOI: 10.1182/blood-2017-05-743187

8 Huang Y, Cui MM, Huang YX, Fu S, Zhang X, Guo H and Wang RT: Preoperative platelet distribution width predicts breast cancer survival. Cancer Biomark 23: 205-211, 2018. PMID: 30198864. DOI: $10.3233 / \mathrm{CBM}-181267$

9 Song Q, Wu JZ, Wang S and Chen WH: Elevated preoperative platelet distribution width predicts poor prognosis in esophageal squamous cell carcinoma. Sci Rep 9: 15234, 2019. PMID: 31645619. DOI: $10.1038 / \mathrm{s} 41598-019-51675-y$
10 Matsunaga T, Saito H, Fukumoto Y, Shimizu S, Kono Y, Murakami Y, Shishido Y, Miyatani K, Yamamoto M, Tokuyasu N, Takano S, Sakamoto T, Honjo S and Fujiwara Y: The postoperative platelet distribution width is useful for predicting the prognosis in patients with esophageal squamous cell carcinoma. Surg Today 50: 123-133, 2020. PMID: 31388825. DOI: 10.1007/s00595-019-01860-3

11 Guo F, Zhu X and Qin X: Platelet distribution width in hepatocellular carcinoma. Med Sci Monit 24: 2518-2523, 2018. PMID: 29689031.

12 Zhang H, Liu L, Fu S, Liu YS, Wang C, Liu T, Liu ZP, Wang RT and Yu KJ: Higher platelet distribution width predicts poor prognosis in laryngeal cancer. Oncotarget 8: 48138-48144, 2017. PMID: 28624815. DOI: 10.18632/oncotarget.18306

13 Zhang X, Cui MM, Fu S, Li LL, Liu YS, Liu ZP, Liu T, Wang RT and $\mathrm{Yu} \mathrm{KJ}$ : Platelet distribution width correlates with prognosis of gastric cancer. Oncotarget 8: 20213-20219, 2017. PMID: 28423627. DOI: 10.18632/oncotarget.15561

14 Cheng S, Han F, Wang Y, Xu Y, Qu T, Ju Y and Lu Z: The red distribution width and the platelet distribution width as prognostic predictors in gastric cancer. BMC Gastroenterol 17: 163, 2017. PMID: 29262773. DOI: 10.1186/s12876-017-0685-7

15 Liu C, Zhang H, Qi Q, Zhang B, Yue D and Wang C: The preoperative platelet distribution width: A predictive factor of the prognosis in patients with non-small cell lung cancer. Thorac Cancer 11: 918-927, 2020. PMID: 32061027. DOI: 10.1111/17597714.13340

16 Cui MM, Li N, Liu X, Yun ZY, Niu Y, Zhang Y, Gao B, Liu T and Wang RT: Platelet distribution width correlates with prognosis of non-small cell lung cancer. Sci Rep 7: 3456, 2017. PMID: 28615714. DOI: 10.1038/s41598-017-03772-z

17 Marnell L, Mold C and Du Clos TW: C-reactive protein: ligands, receptors and role in inflammation. Clin Immunol 117: 104-111, 2005. PMID: 30483265. DOI: 10.3389/fimmu.2018.02607

18 Allin KH and Nordestgaard BG: Elevated C-reactive protein in the diagnosis, prognosis, and cause of cancer. Crit Rev Clin Lab Sci 48: 155-170, 2011. PMID: 22035340. DOI: 10.3109/10408363. 2011.599831

19 Xu HJ, Ma Y, Deng F, Ju WB, Sun XY and Wang H: The prognostic value of C-reactive protein/albumin ratio in human malignancies: an updated meta-analysis. Onco Targets Ther 10: 3059-3070, 2017. PMID: 28790840. DOI: 10.2147/OTT.S137002

20 Brierley J, Gospodarowicz M and Wittekind C: International Union against Cancer. TNM Classification of Malignant Tumors. Eighth Edition. Chichester; Wiley-Blackwell, 2017.

$21 \mathrm{Xu} \mathrm{XL}, \mathrm{Yu} \mathrm{HQ}, \mathrm{Hu} \mathrm{W}$, Song Q and Mao WM: A novel inflammation-based prognostic score, the c-reactive protein/albumin ratio predicts the prognosis of patients with operable esophageal squamous cell carcinoma. PLoS One 10: e0138657, 2015. PMID: 26390126. DOI: 10.1371/journal.pone.0138657

22 Toyokawa T, Kubo N, Tamura T, Sakurai K, Amano R, Tanaka H, Muguruma K, Yashiro M, Hirakawa K and Ohira M: The pretreatment Controlling Nutritional Status (CONUT) score is an independent prognostic factor in patients with resectable thoracic esophageal squamous cell carcinoma: results from a retrospective study. BMC Cancer 16: 722, 2016. PMID: 27599460. DOI: $10.1186 / \mathrm{s} 12885-016-2696-0$

$23 \mathrm{Hu}$ G, Liu G, Ma JY and Hu RJ: Lymphocyte-to-monocyte ratio in esophageal squamous cell carcinoma prognosis. Clin Chim Acta 486: 44-48, 2018. PMID: 30028962. DOI: 10.1016/j.cca. 2018.07.029 
24 Yang Y, Xu H, Zhou L, Deng T, Ning T, Liu R, Zhang L, Wang $\mathrm{X}, \mathrm{Ge} \mathrm{S}, \mathrm{Li} \mathrm{H}$ and $\mathrm{Ba} \mathrm{Y}$ : Platelet to lymphocyte ratio is a predictive marker of prognosis and therapeutic effect of postoperative chemotherapy in non-metastatic esophageal squamous cell carcinoma. Clin Chim Acta 479: 160-165, 2018. PMID: 29325800. DOI: 10.1016/j.cca.2018.01.013

25 Nakatani M, Migita K, Matsumoto S, Wakatsuki K, Ito M, Nakade H, Kunishige T, Kitano M and Kanehiro H: Prognostic significance of the prognostic nutritional index in esophageal cancer patients undergoing neoadjuvant chemotherapy. Dis Esophagus 30: 1-7, 2017. PMID: 28575242. DOI: 10.1093/dote/dox020

26 Wang Y, Chen L, Wu Y, Li P and Che G: The prognostic value of modified Glasgow prognostic score in patients with esophageal squamous cell cancer: a meta-analysis. Nutr Cancer 16: 1-9, 2019. PMID: 31617767. DOI: 10.1080/01635581.2019.1677925

27 Motoyama S, Kitamura M, Saito R, Maruyama K, Sato Y, Hayashi K, Saito H, Minamiya Y and Ogawa J: Surgical outcome of colon interposition by the posterior mediastinal route for thoracic esophageal cancer. Ann Thorac Surg 83: 1273-1278, 2007. PMID: 17383325. DOI: 10.1016/j.athoracsur.2006.11.049

28 Haemmerle M, Stone RL, Menter DG, Afshar-Kharghan V and Sood AK: The platelet lifeline to cancer: challenges and opportunities. Cancer Cell 33: 965-983, 2018. PMID: 29657130. DOI: $10.1016 /$ j.ccell.2018.03.002

29 Zhou S, Ma Y, Shi Y, Tang L, Zheng Z, Fang F, Feng J, Zhang Y, Sun L, Chen Y, Liang B, Jiang S, Shen Z, Yu K and Wang S: Mean platelet volume predicts prognosis in patients with diffuse large B-cell lymphoma. Hematol Oncol 36: 104-109, 2018. PMID: 28736928. DOI: 10.1002/hon.2467

30 Zhuang Q, Xiang L, Xu H, Fang F, Xing C, Liang B, Yu K and Feng J: The independent association of mean platelet volume with overall survival in multiple myeloma. Oncotarget 7: 62640-62646, 2016. PMID: 27566590. DOI: 10.18632/oncotarget.11551
31 Inagaki N, Kibata K, Tamaki T, Shimizu T and Nomura S: Prognostic impact of the mean platelet volume/platelet count ratio in terms of survival in advanced non-small cell lung cancer. Lung Cancer 83: 97-101, 2014. PMID: 24189108. DOI: 10.1016/j.lungcan.2013.08.020

32 Riedl J, Kaider A, Reitter EM, Marosi C, Jäger U, Schwarzinger I, Zielinski C, Pabinger I and Ay C: Association of mean platelet volume with risk of venous thromboembolism and mortality in patients with cancer. Results from the Vienna Cancer and Thrombosis Study (CATS). Thromb Haemost 111: 670-678, 2014. PMID: 24306221. DOI: 10.1160/TH13-07-0603

33 Tamagawa H, Aoyama T, Tamagawa A, Komori K, Maezawa Y, Kano K, Murakawa M, Atsumi Y, Hara K, Kazama K, Numata M, Oshima T, Yukawa N, Masuda M and Rino Y: Influence of the preoperative $\mathrm{C}$-reactive protein-to-albumin ratio on survival and recurrence in patients with esophageal cancer. Anticancer Res 40: 2365-2371, 2020. PMID: 32234939. DOI: 10.21873/anticanres. 14205

34 Katsurahara K, Shiozaki A, Fujiwara H, Konishi H, Kudou M, Shoda K, Arita T, Kosuga T, Morimura R, Murayama Y, Kuriu Y, Ikoma H, Kubota T, Nakanishi M, Okamoto K and Otsuji E: Relationship between postoperative CRP and prognosis in thoracic esophageal squamous cell carcinoma. Anticancer Res 38: 65136518,2018. PMID: 30396980. DOI: 10.21873/anticanres.13016

Received July 26, 2020

Revised August 9, 2020

Accepted August 10, 2020 